Check for updates

Cite this: RSC Adv., 2018, 8, 34291

Received 1st August 2018

Accepted 30th September 2018

DOI: $10.1039 / c 8 r a 06463 f$

rsc.li/rsc-advances

\section{Synthesis of tetraphenylethylene-based conjugated microporous polymers for detection of nitroaromatic explosive compounds $\uparrow$}

\begin{abstract}
Ho Namgung, Jeong Jun Lee, Young Jin Gwon and Taek Seung Lee (DD*
Conjugated microporous polymers (CMPS) containing tetraphenylethylene (TPE) were synthesized via the Suzuki coupling polymerization. The tetrafunctional TPE moiety in the polymer backbone was linked with the difunctional phenylene group to exhibit a porous structure with high fluorescence in the solid state because of aggregation-induced emissive TPE. The porous polymer with a fluorescent TPE group successfully detected nitroaromatic explosive compounds that exhibited fluorescence quenching, in which the polymer shows high quenching efficiency to picric acid among nitroaromatic explosive compounds. The interaction between the electron-rich TPE group and the electron-deficient nitroaromatic compounds played a decisive role in fluorescence quenching via a photoinduced electron transfer (PET). Compared with a linear polymer containing TPE, the porous, crosslinked polymer showed better sensing performance toward nitroaromatic compounds, presumably because of the more efficient interaction between TPE and nitroaromatic compounds in the pores of TPE-based CMP (TPE-CMP).
\end{abstract}

\section{Introduction}

The development of efficient and reliable techniques for the detection of nitroaromatic explosive compounds is becoming an important issue because of the wide use of nitroaromatic compounds as explosive compounds. ${ }^{1,2}$ The detection of nitroaromatic explosive compounds has been investigated using a variety of materials, including organic fluorophores, ${ }^{3-7}$ quantum dots, ${ }^{8,9}$ polymer nanoparticles, ${ }^{10,11}$ metal-organic frameworks, ${ }^{12-16}$ and conjugated polymers. ${ }^{17-20}$ In nitroaromatic explosive compound sensing, fluorescence-based sensors are potentially useful because nitroaromatic explosive compounds can manipulate the emission of fluorophores used, resulting from the electronic changes of the fluorophore,,$^{14,19}$ and various structures of fluorescent probes have been used for detecting explosive compounds. ${ }^{21,22}$ As the fluorescent probe was exposed to nitroaromatic compounds such as TNT, 2,4-dinitrotoluene (2,4-DNT), 2,6-dinitrotoluene (2,6-DNT), and picric acid (PA), the fluorescence was quenched by a PET mechanism. Among the nitroaromatic compounds, PA (2,4,6-trinitrophenol) is known as a highly hazardous and powerful explosive which causing severe human health problems when discharged into our living environments. .,2,23 $^{1,23}$

Organic and Optoelectronic Materials Laboratory, Department of Organic Materials Engineering, Chungnam National University, Daejeon 34134, Korea. E-mail: tslee@ cnu.ac. kr

$\dagger$ Electronic supplementary information (ESI) available. See DOI: 10.1039/c8ra06463f
Conjugated microporous polymers (CMPs) are synthesized from multifunctional organic monomers to obtain the porous structures, in which crosslinked, delocalized conjugated structures exhibit high fluorescence, porosity, a large specific surface area, and good physical and chemical stability. ${ }^{24,25}$ Based on these properties, CMPs have been studied in versatile applications, including storage, ${ }^{26-29}$ catalysis, $^{30}$ optoelectricity, ${ }^{31}$ and energy harvesting. ${ }^{32}$

Conventional fluorescent molecules generally are subjected to decreased emission in the solid state because of the phenomenon of aggregation-caused quenching (ACQ). Some fluorophores, such as TPE, also show the opposite phenomenon of aggregation-induced emission (AIE), exhibiting high fluorescence in the solid state. ${ }^{33-37}$ Thus, it is expected that the introduction of TPE into CMPs would provide them with fluorescent properties for versatile applications, even in the solid state. There are several reports on specific applications of TPE-based CMPs in color-tuning ${ }^{38}$ and supercapacitors. ${ }^{31}$ These investigations have revealed the advantages of CMPs integrated with AIE. Thus, we focused on the porous structure of TPE-CMP, in which the pores would be helpful for interactions with nitroaromatic explosive compounds and, as a result, the fluorescence of TPE-based CMP (TPE-CMP) would be effectively altered. Hence, CMP-based fluorescent sensors were prepared for the detection of nitroaromatic explosives such as PA. When exposed to nitroaromatic explosive compounds, the fluorescence was immediately quenched because of PET between the electron-deficient nitroaromatic explosives and the electron-rich TPE-CMP. The sensing efficiency was improved over that of the linear counterpart, indicating the importance of the porous structure. 


\section{Experimental}

\section{Instruments and materials}

${ }^{1} \mathrm{H}$ NMR spectra were obtained on a Bruker Fourier-300 spectrometer and the solid-state NMR spectrum was obtained on a Bruker DSX-300 solid-state FT-NMR spectrometer (Korea Basic Science Institute). Thermogravimetric analysis (TGA) was performed with a Mettler-Toledo TGA/DSC analyzer. FT-IR spectra were obtained on a Bruker Tensor 27 spectrometer. UV-vis absorption spectra were recorded on a PerkinElmer Lambda 35 spectrometer. Photoluminescence spectra were taken using a Varian Cary Eclipse spectrometer. Scanning electron microscopy (SEM) images were obtained using a Hitachi S-4800 instrument. Nitrogen sorption isotherms were obtained on a micromeritics model ASAP 2010 analyzer. Before the measurement, the polymer was degassed in a vacuum at $573 \mathrm{~K}$ for $10 \mathrm{~h}$. The Brunauer-Emmett-Teller (BET) method was used to obtain the specific surface area of the sample. All chemicals were purchased from Sigma-Aldrich (USA) and solvents were purchased from Samchun Chemicals (Korea). All reagents were used without further purification unless otherwise noted.

\section{Synthesis of 1,1,2,2-tetrakis(4-bromophenyl)ethene (2)}

$1(1 \mathrm{~g}, 3.01 \mathrm{mmol})$ was dissolved in chloroform $(20 \mathrm{~mL})$ at room temperature. The temperature of the solution was cooled to $0{ }^{\circ} \mathrm{C}$ using an ice bath. After cooling, bromine $(1.12 \mathrm{~mL}, 21.07 \mathrm{mmol})$ was added slowly. The mixture was stirred at $0{ }^{\circ} \mathrm{C}$ for $7 \mathrm{~h}$. After the reaction, the mixture was heated to room temperature. A sodium bisulfate solution was added to remove the remaining bromine. The mixture was dissolved in methylene chloride and washed with water $(100 \mathrm{~mL})$ three times. The combined organic layer obtained was dried on $\mathrm{MgSO}_{4}$. After the evaporation of methylene chloride, the product was recrystallized in $n$-hexane, and a white powder was obtained (yield $1.49 \mathrm{~g}, 77 \%) .{ }^{1} \mathrm{H}$ NMR $\left(300 \mathrm{MHz} \mathrm{CDCl}_{3}\right) 7.2(8 \mathrm{H}, \mathrm{m}), 6.8(8 \mathrm{H}, \mathrm{m})$ ppm. FT-IR $(\mathrm{KBr}$ pellet, $\mathrm{cm}^{-1}$ ): $3005(\mathrm{C}-\mathrm{H})$, 1585-1394 $(\mathrm{C}=\mathrm{C}), 1008$ (C-Br).

\section{Synthesis of TPE-CMP}

A molar ratio of $1: 2(2: 3)$ is depicted here. $2(0.80 \mathrm{~g}, 1.23$ $\mathrm{mmol}$ ) and 1,4-benzenediboronic acid bis(pinacol)ester (3) $(0.812 \mathrm{~g}, 2.46 \mathrm{mmol})$ were dissolved in a mixture of toluene containing an aqueous $2 \mathrm{M}$ potassium carbonate solution (5 $\mathrm{mL}$ ) under argon atmosphere. After addition of tetrakis(triphenylphosphine)palladium(0) $(0.071 \mathrm{~g}, 0.062 \mathrm{mmol})$, the reaction mixture was stirred at $100{ }^{\circ} \mathrm{C}$ for $24 \mathrm{~h}$. After reaction, the mixture was cooled and added to methanol $(300 \mathrm{~mL})$ and the precipitate was isolated by filtration. The precipitate was washed with water and acetone. The product was purified with tetrahydrofuran (THF), methylene chloride, and chloroform for each 1 day in a Soxhlet apparatus. After drying under vacuum, TPE-CMP was obtained (yield $0.37 \mathrm{~g}, 74 \%$ ). Solid ${ }^{13} \mathrm{C}$ NMR (ppm): 210-190 (m), 138-125 (m), 79-55 (m), 22.53 (s) ppm. FTIR (KBr pellet, $\mathrm{cm}^{-1}$ ): $3024(\mathrm{C}-\mathrm{H}), 1598-1388(\mathrm{C}=\mathrm{C}), 1003(\mathrm{C}-$ $\mathrm{Br}$ ). Anal calcd. for $\mathrm{C}_{38} \mathrm{H}_{24}$ : C, 94.97\%; H, 5.03\%. Found. C, $94.54 \%$; H, 5.32\%.

\section{Synthesis of TPE-Ph}

1,2-Bis(4-bromophenyl)-1,2-diphenylethene (0.603 g, 1.23 $\mathrm{mmol})$ and $3(0.406 \mathrm{~g}, 1.23 \mathrm{mmol})$ were dissolved in a mixture of toluene containing aqueous $2 \mathrm{M}$ potassium carbonate solution $(5 \mathrm{~mL})$ under an argon atmosphere. The same procedure that was employed for TPE-CMP was used. After reaction, the mixture was cooled and added to methanol $(300 \mathrm{~mL})$ and the precipitate was isolated by filtration. The precipitate was washed with methanol and acetone. After drying under vacuum, TPE-Ph was obtained (yield $0.33 \mathrm{~g}, 66 \%) .{ }^{1} \mathrm{H}$ NMR $(300 \mathrm{MHz}$, $\left.\mathrm{CDCl}_{3}\right) 7.61$ (4H, d), 7.41 (4H, m), $7.13(14 \mathrm{H}, \mathrm{s})$ ppm. FT-IR (KBr pellet, $\mathrm{cm}^{-1}$ ): 3051-3024 (C-H), 1598-1359 (C=C), $1004(\mathrm{C}-\mathrm{Br})$. Anal calcd. for $\mathrm{C}_{32} \mathrm{H}_{22}$ : C, 94.55\%; H, 5.45\%. Found. C, 93.13\%; $\mathrm{H}, 6.71 \%$.

\section{Detection of explosive compounds}

TPE-CMP was suspended in methylene chloride $(0.375 \mathrm{mg}$ $\mathrm{mL}^{-1}$ ). Explosive compounds such as 2,4-DNT, 2,6-DNT, PA, and 2,3-dinitro-2,3-dimethylethane (2,3-DN-2,3-DM) were all dissolved in methylene chloride. Each solution of the explosive simulant was added to the TPE-CMP suspension of and the change in fluorescence was recorded on a fluorescence spectrometer. Fluorescence was measured with a $1 \mathrm{~cm}$ quartz cuvette containing the TPE-CMPs dispersion. The widths of the excitation and the emission silts were both $5 \mathrm{~nm}$.

\section{Results and discussion}

Compounds 1 and $\mathbf{2}$ were synthesized according to a previously published method. ${ }^{39}$ The synthetic route to TPE-CMP is illustrated in Scheme 1, where TPE-CMP was prepared via a Suzuki
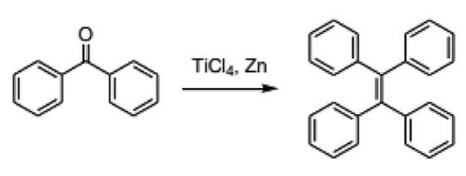

1

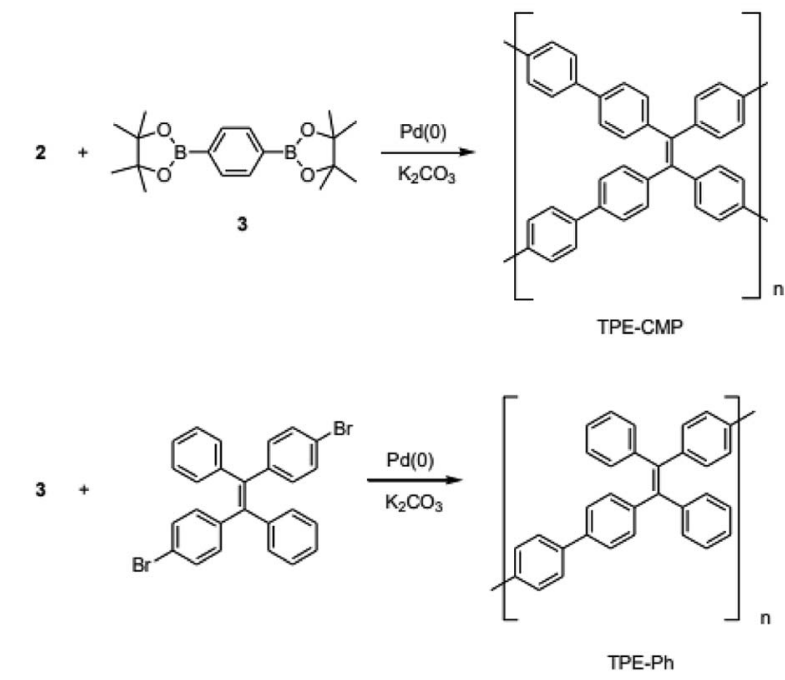

Scheme 1 Synthesis of polymers. 
coupling reaction between 2 and 3 using feed ratios of $1: 1$, $1: 2$, and $1: 3$. The TPE-CMPs were insoluble in organic solvents, such as acetone, methanol, ethanol, THF, DMF, and chloroform, indicative of the formation of a crosslinked structure. The chemical structure of TPE-CMP was confirmed by solid-state ${ }^{13} \mathrm{C}$ NMR, FT-IR, and elemental analysis. The porosity of the TPE-CMPs was analyzed by nitrogen adsorption isotherms, and the specific surface area $\left(a_{\mathrm{s}, \mathrm{BET}}\right)$, total pore volume, and average pore size of the polymers were calculated using the BET equation (Table 1). The TPE-CMP prepared with the 1:2 monomer ratio exhibited the highest specific surface area and pore volume and, thus the TPE-CMP with the molar ratio of $1: 2$ was used for subsequent experiments. The nitrogen gas adsorption isotherms of the TPE-CMP with $1: 2$ ratio showed type I physisorption isotherms with a steep increase in gas uptake in the lower $P / P_{\mathrm{o}}$ region $(<0.05)$, indicative of a permanent microporous structure with a pore diameter between 2 and $50 \mathrm{~nm}$ (Fig. 1).40,41 The TGA thermogram shows that the TPE-CMP did not significantly decompose up to $400{ }^{\circ} \mathrm{C}$ and $86 \%$ of the compound remained even at $500{ }^{\circ} \mathrm{C}$ (Fig. 2a). TPE-CMP exhibited an irregularly shaped morphology in SEM images (Fig. 2b).

TPE is well known for its AIE, and thus monomer 2 showed strong fluorescence in the solid state. Solid 2 showed a UV-vis

Table 1 BET data of TPE-CMPs

\begin{tabular}{lccc}
\hline Molar ratio $(2: 3)$ in feed & $1: 1$ & $1: 2$ & $1: 3$ \\
\hline Specific surface area, $a_{\mathrm{s}, \mathrm{BET}}\left[\mathrm{m}^{2} \mathrm{~g}^{-1}\right]$ & 20.40 & 680.57 & 599.64 \\
Total pore volume $\left[\mathrm{cm}^{3} \mathrm{~g}^{-1}\right]$ & 0.026 & 0.403 & 0.468 \\
Average pore diameter $[\mathrm{nm}]$ & 5.15 & 2.37 & 3.12
\end{tabular}

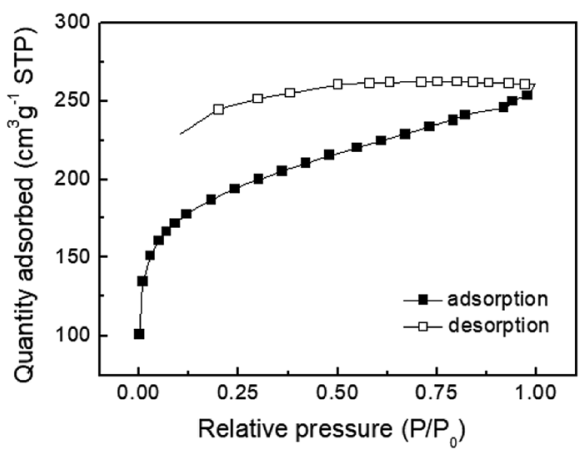

Fig. 1 Nitrogen gas adsorption isotherm of TPE-CMP at $77 \mathrm{~K}$.
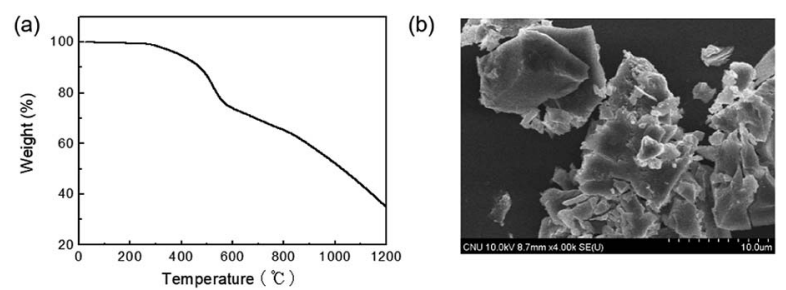

Fig. 2 (a) TGA thermogram and (b) SEM image of TPE-CMP. absorption at $330 \mathrm{~nm}$ and strong fluorescence at $480 \mathrm{~nm}$ (Fig. 3a). TPE-CMP containing 2 exhibited a strong emission at $550 \mathrm{~nm}$ and excitation was observed at $330 \mathrm{~nm}$, indicating that the absorption was dependent on the TPE unit. The absolute quantum yield of TPE-CMP was $32 \%$. Such a red shift in the fluorescence of TPE-CMP compared with 2 resulted from the extended conjugation of the polymer backbone structure. The blue emission of 2 and the yellow fluorescence of TPE-CMP could be observed by the naked eye (Fig. 3b). The UV-vis absorption spectrum of TPE-CMP in solution could not be obtained because of the difficulty in sample preparation (the compounds are not soluble in any solvent and a transparent solid sample is not easy to prepare).

The detection of explosive compounds, namely 2,4-DNT, 2,6DNT, PA, and 2,3-DN-2,3-DM was investigated in terms of fluorescence change in TPE-CMP, in which the nitroaromatics acted as fluorescence quenchers via the PET mechanism (Scheme 2). Upon exposure of the TPE-CMP dispersion to the explosive compounds, an excited electron of TPE-CMP was transferred to the explosive compounds. The fluorescence was then quenched immediately through PET between the TPE moieties and the explosive compounds (inset photographs in Scheme 2). Among the explosive compounds investigated, the nitroaromatic compounds including 2,4-DNT, 2,6-DNT, and PA efficiently quenched the fluorescence of TPE-CMP, whereas the non-nitroaromatic 2,3-DN-2,3-DM showed a negligible effect on this property (Fig. 4). As soon as 2,4-DNT, 2,6-DNT, and PA were exposed to TPE-CMP, the fluorescence was quenched. Such a decrease in the fluorescence was caused by the aromatic structures of 2,4-DNT, 2,6-DNT, and PA, which promoted absorption of these explosives through the $\pi-\pi$ interaction. This can be more clearly observed in the case of PA, in which almost all the fluorescence was quenched based on the relative fluorescence intensity ratios. Among the three explosives, PA exhibited effective fluorescence quenching (the quenching (a)

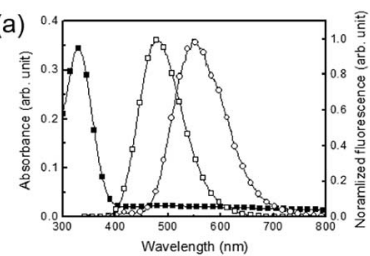

(b)

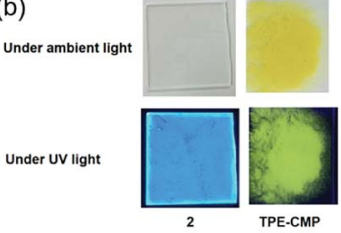

Fig. 3 (a) Absorption ( $\square$ ) and fluorescence spectra ( $\square$ ) of solid 2 and fluorescence spectrum (O) of TPE-CMP, excitation wavelength $330 \mathrm{~nm}$. (b) Photographs of 2 and TPE-CMP under ambient and UV lights (365 nm).
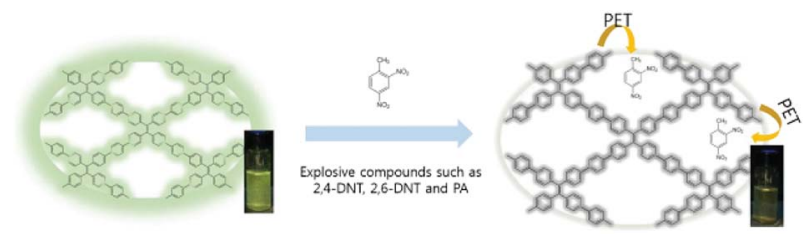

Scheme 2 Fluorescence quenching of TPE-CMP in the presence of nitroaromatic compounds via PET. 
(a)

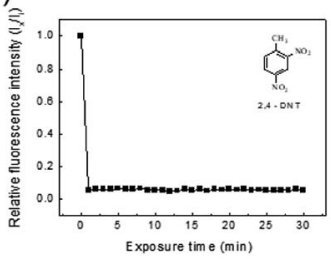

(c)

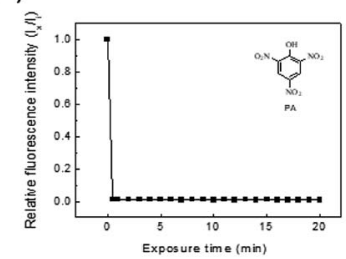

(b)

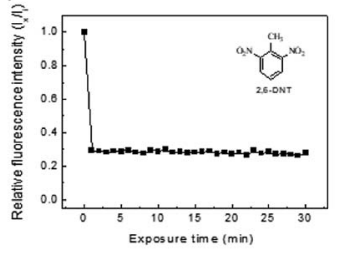

(d)

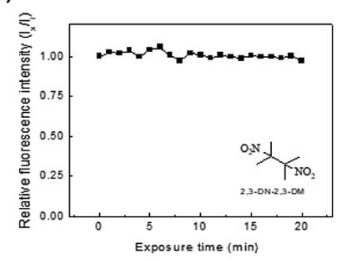

Fig. 4 Changes in the relative fluorescence intensities $\left(I_{x} / I_{\mathrm{i}}\right)$ of $1: 2$ TPE-CMP $\left(0.375 \mathrm{mg} \mathrm{mL}^{-1}\right.$ in $\left.\mathrm{CH}_{2} \mathrm{Cl}_{2}\right)$ upon various exposure time in the presence of (a) 2,4-DNT, (b) 2,6-DNT, (c) PA, and (d) 2,3-DN-2,3DM. [Explosives] $=2.5 \times 10^{-3} \mathrm{M}$. Excitation wavelength $330 \mathrm{~nm} . /_{\mathrm{i}}$ and $I_{x}$ represent fluorescence intensity at $550 \mathrm{~nm}$ in the absence and presence of explosives, respectively.
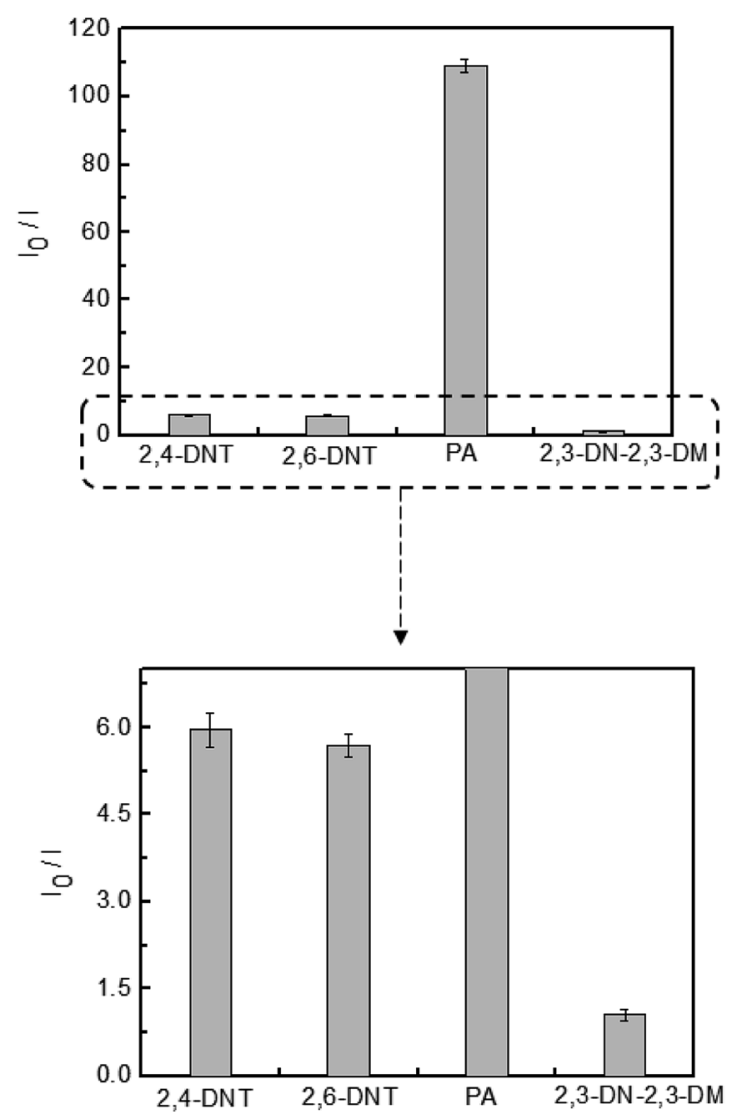

Fig. 5 Relative changes in fluorescence intensity of TPE-CMP (0.375 mg mL $\mathrm{mb}^{-1} \mathrm{CH}_{2} \mathrm{Cl}_{2}$ ) upon exposure to 2,4-DNT, 2,6-DNT, PA, and 2,3-DN-2,3-DM. [Explosives] $=2.5 \times 10^{-3} \mathrm{M}$. Excitation wavelength $330 \mathrm{~nm}$. $I_{0}$ and $/$ represent fluorescence intensity at $550 \mathrm{~nm}$ before and after exposure to explosives, respectively.

efficiency improved by a factor of more than 10), presumably because of the lower energy of the lowest unoccupied molecular orbital (LUMO) of PA than other explosive resulting in facile electron transfer (Fig. 5). ${ }^{14}$

A less efficient quenching of TPE-CMP than PA was observed upon exposure to 2,4-DNT and 2,6-DNT. The fluorescence of TPE-CMP was gradually decreased with increase in the concentration of nitroaromatic compounds (Fig. 6), resulting in the maximum quenching with approximately $2.5 \times 10^{-3} \mathrm{M}$ of each nitroaromatic compound. This can be explained by the Stern-Volmer equation: ${ }^{20}$

$$
F_{\mathrm{o}} / F=1+K_{\mathrm{SV}}[\mathrm{Q}]
$$

The equation provides a quantitative relationship between the changes in fluorescence intensity $\left(F_{\mathrm{o}} / F\right)$ and the concentration of added nitroaromatic compounds ([Q]). The slope of the plot is the Stern-Volmer constant $\left(K_{\mathrm{SV}}\right)$. The $K_{\mathrm{SV}}$ values for 2,4DNT, 2,6-DNT, and PA were estimated as $1.84 \times 10^{3} \mathrm{M}^{-1}, 1.70 \times$ $10^{3} \mathrm{M}^{-1}$, and $1.19 \times 10^{4} \mathrm{M}^{-1}$, indicating that the PA could be most sensitively detected, in accordance with the result from Fig. 5 For comparison, monomer 2 in methylene chloride showed $\sim 5.0 \times 10^{2} \mathrm{M}^{-1}$ of $K_{\mathrm{SV}}$ toward PA, indicative of a relatively low sensitivity.

For further elucidation of the uptake of PA by porous TPECMP, FT-IR spectroscopy was used to investigate whether the PA was present in TPE-CMP; the FT-IR sample was obtained after separation of TPE-CMP from the PA solution by centrifugation at $12000 \mathrm{rpm}$ (Fig. 7). The characteristic bands

(a)
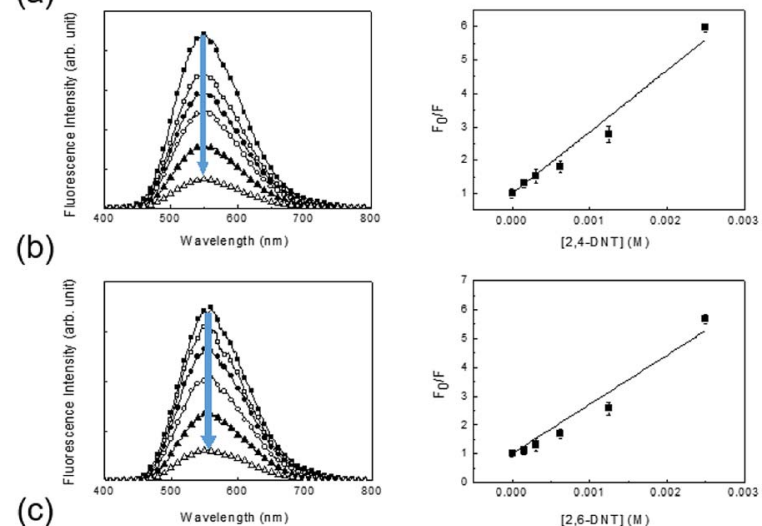

(b)
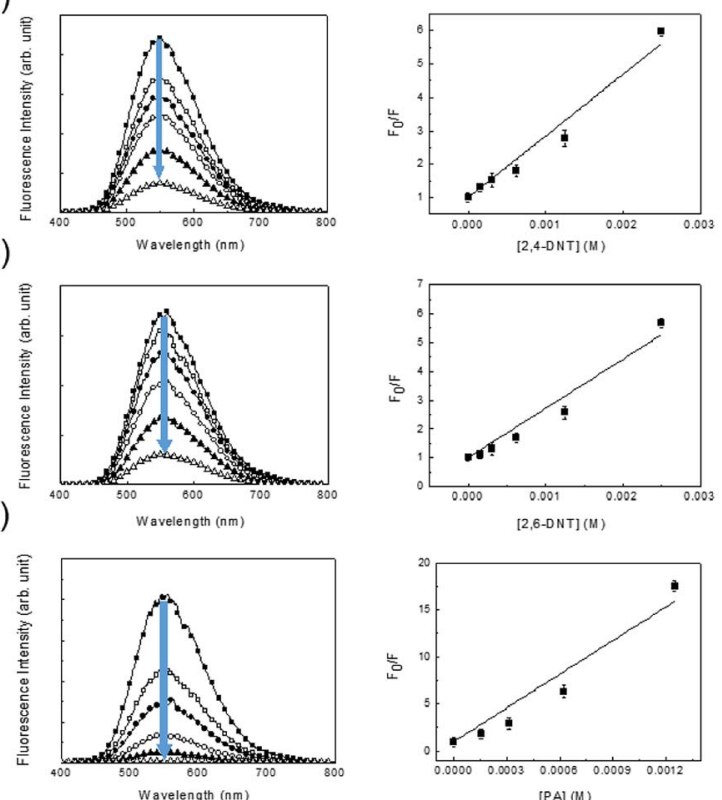

Fig. 6 Changes in the fluorescence of TPE-CMP $\left(0.375 \mathrm{mg} \mathrm{mL}^{-1}\right.$ in $\mathrm{CH}_{2} \mathrm{Cl}_{2}$ ) with various concentrations of 2,4-DNT (a); 2,6-DNT (b); PA (c). Exposure time $5 \mathrm{~min}$. [Explosives] $=0 ; 1.56 \times 10^{-4} ; 3.13 \times 10^{-4}$; $6.25 \times 10^{-4} ; 1.25 \times 10^{-3} ; 2.5 \times 10^{-3} \mathrm{M}$. Excitation wavelength $330 \mathrm{~nm}$. Right figures: Stern-Volmer plots of the fluorimetric titration. $F_{\circ}$ and $F$ represent fluorescent intensity at $550 \mathrm{~nm}$ before and after exposure to explosives, respectively. 


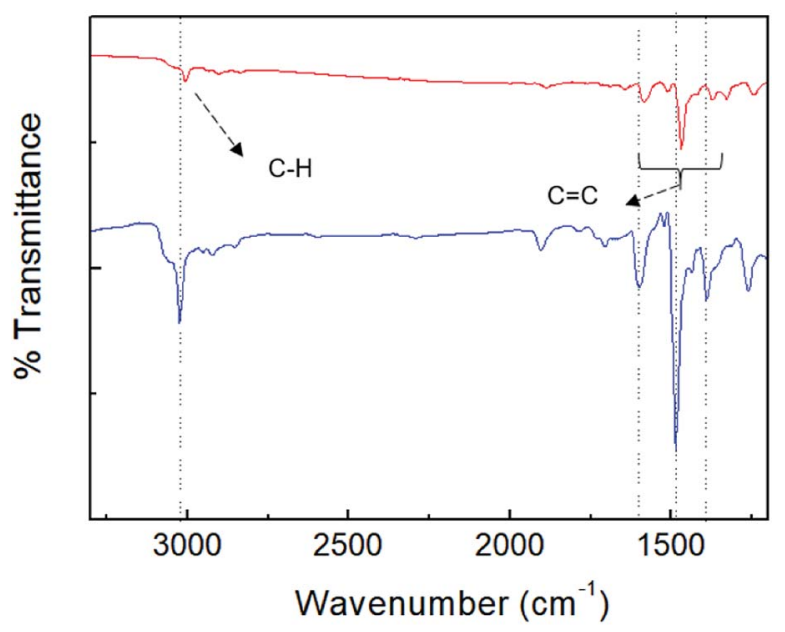

Fig. 7 FT-IR spectra of TPE-CMP before (blue) and after (red) exposure to PA.

corresponding to TPE-CMP including $\mathrm{C}=\mathrm{C}$ and $\mathrm{C}-\mathrm{C}$ bonds were shifted to a lower wavenumber by $\sim 20 \mathrm{~cm}^{-1}$ because of the weakening of such bonds by $\pi-\pi$ interactions between the nitroaromatic compounds and TPE-CMP, indicating the presence of the nitroaromatic compounds in the pores of TPE-CMP. To elucidate the effect of the porous structure on the PET, a TPE-containing linear polymer (TPE-Ph) was synthesized to obtain the nonporous polymer TPE-Ph (Scheme 1). The TPE-Ph was responsive to PA with a $K_{\mathrm{Sv}}$ of $3.53 \times 10^{2} \mathrm{M}^{-1}$, which showed less effective fluorescence quenching compared with TPE-CMP, indicating that the porosity could enhance the sensing ability of nitroaromatic compounds via an increased interaction between TPE and the nitroaromatic compounds in the pores.

\section{Conclusions}

We synthesized TPE-CMPs via a Suzuki coupling reaction between the TPE-based AIE monomer and diboronic ester. The synthesized TPE-CMPs could be used as a selective sensor for PA. The porous, crosslinked TPE-CMPs were insoluble in organic solvents and exhibited excellent thermal stability. Because of the presence of an AIE-type unit, TPE, the TPE-CMP exhibited strong fluorescence in the solid state, which was beneficial for the use of TPE-CMP in solid-state sensing. Upon exposure of TPE-CMP to nitroaromatic compounds, such as 2,4DNT, 2,6-DNT, and PA, the fluorescence was immediately quenched because PET took place between the nitroaromatics and the TPE moieties of TPE-CMP. In terms of the Stern-Volmer constant, the TPE-CMP was more sensitive for detecting PA than for other nitroaromatics. These results confirmed that TPECMP can be useful for detecting nitroaromatic explosive compounds through efficient fluorescence quenching.

\section{Conflicts of interest}

There are no conflicts to declare.

\section{Acknowledgements}

Financial support from the National Research Foundation (NRF) of Korean government through Basic Science Research Program (2018R1A2A2A14022019) is gratefully acknowledged.

\section{Notes and references}

1 Z. Hu, B. J. Deibert and J. Li, Chem. Soc. Rev., 2014, 43, 58155840 .

2 J. Ye, L. Zhao, R. F. Bogale, Y. Gao, X. Wang, X. Qian, S. Guo, J. Zhao and G. Ning, Chem.-Eur. J., 2015, 21, 2029-2037.

3 J. Wu, B. Kwon, W. Liu, E. V. Anslyn, P. Wang and J. S. Kim, Chem. Rev., 2015, 115, 7893-7943.

4 B. Gogoi and N. S. Sarma, ACS Appl. Mater. Interfaces, 2015, 7, 11195-11202.

5 T. Liu, L. Ding, K. Zhao, W. Wang and Y. Fang, J. Mater. Chem., 2012, 22, 1069-1077.

6 S. Sandhu, R. Kumar, P. Singh, A. Mahajan, M. Kaur and S. Kumar, ACS Appl. Mater. Interfaces, 2015, 7, 10491-10500. 7 Z. Zhang, S. Chen, R. Shi, J. Ji, D. Wang, S. Jin, T. Han, C. Zhou and Q. Shu, Talanta, 2017, 166, 228-233.

8 A. H. Malik, S. Hussain, A. Kalita and P. K. Iyer, ACS Appl. Mater. Interfaces, 2015, 7, 26968-26976.

9 A. Pal, M. P. Sk and A. Chattopadhyay, ACS Appl. Mater. Interfaces, 2016, 8, 5758-5762.

10 S. Shanmugaraju, C. Dabadie, K. Byrne, A. J. Savyasachi, D. Umadevi, W. Schmitt, J. A. Kitchen and T. Gunnlaugsson, Chem. Sci., 2017, 8, 1535-1546.

11 A. S. Tanwar, S. Hussain, A. H. Malik, M. A. Afroz and P. K. Iyer, ACS Sens., 2016, 1, 1070-1077.

12 S. Sarkar, S. Dutta, S. Chakrabarti, P. Bairi and T. Pal, ACS Appl. Mater. Interfaces, 2014, 6, 6308-6316.

13 C. Zhang, Y. Yan, Q. Pan, L. Sun, H. He, Y. Liu, Z. Liang and J. Li, Dalton Trans., 2015, 44, 13340-13346.

14 Y. Hu, M. Ding, X. Q. Liu, L. B. Sun and H. L. Jiang, Chem. Commun., 2016, 52, 5734-5737.

15 W. P. Lustig, S. Mukherjee, N. D. Rudd, A. V. Desai, J. Li and S. K. Ghosh, Chem. Soc. Rev., 2017, 46, 3242-3285.

16 S. S. Nagarkar, A. V. Desai and S. K. Ghosh, CrystEngComm, 2016, 18, 2994-3007.

17 T. M. Swager, Acc. Chem. Res., 1998, 31, 201-207.

18 H. Nie, Y. Zhao, M. Zhang, Y. Ma, M. Baumgarten and K. Müllen, Chem. Commun., 2011, 47, 1234-1236.

19 P. Anzenbacher Jr, L. Mosca, M. A. Palacios, G. V. Zyryanov and P. Koutnik, Chem.-Eur. J., 2012, 18, 12712-12718.

20 T. H. Kim, H. J. Kim, C. G. Kwak, W. H. Park and T. S. Lee, J. Polym. Sci., Part A: Polym. Chem., 2006, 44, 2059-2068.

21 L. Mosca, S. K. Behzad and P. Anzenbacher Jr, J. Am. Chem. Soc., 2015, 137, 7967-7969.

22 W. Wu, S. Ye, R. Tang, L. Huang, Q. Li, G. Yu, Y. Liu, J. Qin and Z. Li, Polymer, 2012, 53, 3163-3171.

23 K. M. Wollin and H. H. Dieter, Arch. Environ. Contam. Toxicol., 2005, 49, 18-26.

24 J. X. Jiang, F. Su, A. Trewin, C. D. Wood, N. L. Campbell, H. Niu, C. Dickinson, A. Y. Ganin, M. J. Rosseinsky, 
Y. Z. Khimyak and A. I. Cooper, Angew. Chem., Int. Ed., 2007, 46, 8574-8578.

25 Y. Xu, S. Jin, H. Xu, A. Nagai and D. Jiang, Chem. Soc. Rev., 2013, 42, 8012-8031.

26 R. Dawson, E. Stöckel, J. R. Holst, D. J. Adams and A. I. Cooper, Energy Environ. Sci., 2011, 4, 4239-4245.

27 Q. Chen, J.-X. Wang, Q. Wang, N. Bian, Z.-H. Li, C.-G. Yan and B.-H. Han, Macromolecules, 2011, 44, 7987-7993.

28 C. Zhang, L.-H. Peng, B. Li, Y. Liu, P.-C. Zhu, Z. Wang, D.-H. Zhan, B. Tan, X.-L. Yang and H.-B. Xu, Polym. Chem., 2013, 4, 3663-3666.

29 J. Park and C. Y. Lee, Polymer, 2018, 42, 371-376.

30 P. Zhang, Z. Weng, J. Guo and C. Wang, Chem. Mater., 2011, 23, 5243-5249.

31 K. Yuan, P. Guo-Wang, T. Hu, L. Shi, R. Zeng, M. Forster, T. Pichler, Y. Chen and U. Scherf, Chem. Mater., 2015, 27, 7403-7411.

32 L. Chen, Y. Honsho, S. Seki and D. Jiang, J. Am. Chem. Soc., 2010, 132, 6742-6748.
33 Y. Hong, J. W. Lam and B. Z. Tang, Chem. Soc. Rev., 2011, 40, 5361-5388.

34 Y. Hong, J. W. Lam and B. Z. Tang, Chem. Commun., 2009, 29, 4332-4353.

35 L. Yan, Y. Zhang, B. Xu and W. Tian, Nanoscale, 2016, 8, 2471-2487.

36 C. Zhang, S. Jin, S. Li, X. Xue, J. Liu, Y. Huang, Y. Jiang, W.-Q. Chen, G. Zou and X.-J. Liang, ACS Appl. Mater. Interfaces, 2014, 6, 5212-5220.

37 E. Zhao, Y. Chen, H. Wang, S. Chen, J. W. Lam, C. W. Leung, Y. Hong and B. Z. Tang, ACS Appl. Mater. Interfaces, 2015, 7, 7180-7188.

38 Y. Xu, A. Nagai and D. Jiang, Chem. Commun., 2013, 49, 1591-1593.

39 N. B. Shustova, B. D. McCarthy and M. Dinca, J. Am. Chem. Soc., 2011, 133, 20126-20129.

40 M. Khalfaoui, S. Knani, M. Hachicha and A. B. Lamine, J. Colloid Interface Sci., 2003, 263, 350-356.

41 K. S. Sing, Pure Appl. Chem., 1985, 57, 603-619. 